\title{
TITLE:
}

\section{Automatic GCP Extraction of Fully Polarimetric SAR Images}

$\operatorname{AUTHOR}(\mathrm{S})$ :

Chureesampant, Kamolratn; Susaki, Junichi

\section{CITATION:}

Chureesampant, Kamolratn ... [et al]. Automatic GCP Extraction of Fully Polarimetric SAR Images. IEEE Transactions on Geoscience and Remote Sensing 2014, 52(1): 137-148

\section{ISSUE DATE:}

2014-01

URL:

http://hdl.handle.net/2433/193300

\section{RIGHT:}

(c) 2014 IEEE. Personal use of this material is permitted. Permission from IEEE must be obtained for all other uses, in any current or future media, including reprinting/republishing this material for advertising or promotional purposes, creating new collective works, for resale or redistribution to servers or lists, or reuse of any copyrighted component of this work in other works.; この論文は出版社版でありません。引用の際には出版社版をご確認ご利用ください。; This is not the published version. Please cite only the published version. 


\title{
Automatic GCP Extraction of Fully Polarimetric SAR Images
}

\author{
Kamolratn Chureesampant and Junichi Susaki, Member, IEEE
}

\begin{abstract}
This paper presents a method for automatic extraction of Ground Control Points (GCPs) of fully polarimetric Synthetic Aperture Radar (SAR) images obtained from various satellites with different viewing angles. The Scale-Invariant Feature Transform (SIFT) algorithm is applied to extract candidate GCPs, where two-way keypoint matching eliminates improbable correspondence keypoints. Minimizing the Root Mean Squared Error (RMSE) also removes matching points with large RMSE through a pseudo-affine transformation. In addition, information entropy and spatial dispersion quality constraints enable quantification of the spatial distribution of the GCPs. In accordance with full polarization, applying the SIFT-OCT algorithm (the SIFT algorithm with the first scale-space octave skipped) to polarimetric SAR data is examined. The Total Power (TP) image represents a combination of the characteristic of all four polarization images ( $\mathrm{HH}, \mathrm{HV}, \mathrm{VH}$, and VV). Therefore, GCP extraction using TP image rather than each polarization image is proposed in order to maximize the accuracy of GCP extraction for all of the polarization data, as the TP image generates the highest Signal-to-Noise Ratio (SNR) value. The SNR in conjunction with the Matching Correlation Surface (MCS) is used as an indicator of the reliability and accuracy of GCP extraction. After successfully applying the method to Advanced Land Observing Satellite (ALOS)/Phased Array type L-band Synthetic Aperture Radar (PALSAR) and Japanese Earth Resources Satellite-1 (JERS-1) SAR images, the GCP matching accuracy is further improved by using geometric calibration.
\end{abstract}

Index Terms-Automatic GCP extraction, polarimetric Synthetic Aperture Radar (SAR), Total Power (TP), Scale-Invariant Feature Transform (SIFT), geometric calibration, Signal-to-Noise Ratio (SNR).

Manuscript received October 3, 2010; revised March 31, 2011, July 31, 2011 and November 20, 2011. This work was supported in part by a program of the 3rd ALOS Research Announcement, the Japan Aerospace Exploration Agency (JAXA), and The Okawa Foundation for Information and Telecommunications.

K. Chureesampant is with the Geoinformatics Laboratory, Department of Urban and Environmental Engineering, Graduate School of Engineering, Kyoto University, Kyoto, Japan (e-mail: kamolratn.c@ft5.ecs.kyoto-u.ac.jp).

J. Susaki is with the Geoinformatics Laboratory, Department of Civil and Earth Resources Engineering, Graduate School of Engineering, Kyoto University, Kyoto, Japan (e-mail: susaki.junichi.3r@kyoto-u.ac.jp). 


\section{INTRODUCTION}

In recent years, the operation of fully polarimetric Synthetic Aperture Radar (SAR) systems has accelerated the development of various applications, for example, image fusion and classification or detection of changes in images. Prior to implementing such applications, SAR images must be co-registered with sufficient accuracy depending on the application requirements [1], [2]. The Ground Control Point (GCP) extraction is the most important first step in an accurate image co-registration. The principles of ideal GCP extraction are as follows: (1) the GCPs should be spread uniformly throughout the image to provide a clear indication of distortion in any direction; (2) the minimum number of the GCPs should be three for possible linear solution; (3) their coordinates should be precisely known. GCP extraction can be performed manually or automatically. For manual extraction, GCPs are determined by visual examination, which is a time-consuming task with potentially unstable results [3]. GCP extraction for SAR images is more difficult than for optical remote sensing images due to the influence of multiplicative speckle noise [1], [2], and no general mapping exists between the intensities of different SAR images [4]. For these reasons, it is extremely challenging to attain high precision in determining GCPs from images as well as high accuracy and reliability when performing co-registration.

This process is additionally hindered by the GCP extraction of polarimetric SAR images since each type of polarization (HH, $\mathrm{HV}, \mathrm{VH}$ or VV) yields a different pattern of scattering from the ground. Also, the number and locations of GCPs extracted from pairs of HH images may be different from the results extracted from pairs of images with other polarization patterns. In addition, polarimetric SARs operate in several types of observation modes. For example, Advanced Land Observing Satellite (ALOS)/Phased Array type L-band Synthetic Aperture Radar (PALSAR) operates in a fine beam single polarization (FBS) mode, fine beam dual polarization (FBD) mode, fine beam fully polarimetric (PLR) mode [5] and ScanSAR mode. Whereas the off-nadir angle in the PLR mode is $21.5^{\circ}$, the angles for the FBS and FBD modes are set to $34.3^{\circ}$. Therefore, if images acquired, for example, in PLR and FBS modes are co-registered, the effects of the different acquisition viewing geometries in these two modes may become apparent. This problem may also occur when other types of SAR images acquired at different viewing geometries are co-registered. The effects of such differences in viewpoint and viewing geometry should be investigated for the purposes of improving the accuracy of GCP extraction. Since the motivation for this paper is the development of a method for accurate automatic GCP extraction of SAR images, using fully polarimetric synthetic aperture radar (PolSAR) images, it also extends to include the GCP extraction using two or more images acquired at different times, from different sensors or from different viewpoints.

According to the literature, the GCP extraction is performed primarily using feature based techniques. These techniques are very sensitive to effects such as multiplicative speckle in the SAR images [2]; and it may be difficult to extract and match features (i.e., shapes) that change with time [1]. As a matter of fact, such techniques for GCP extraction are bound to be uncertain. Therefore, the 
point feature-based multi-sensor SAR image matching should be considered.

The Scale-Invariant Feature Transform (SIFT) algorithm proposed by Lowe [6] can be used to solve problems arising from these difficulties since it constitutes an approach for detecting and extracting local feature descriptors that are reasonably invariant to changes in illumination, image noise, rotation, scaling and small changes in viewpoint. Schwind et al. [1] stated that the SIFT algorithm is capable of selecting and matching different characteristic features between images at various levels of scaling. With these properties, the SIFT algorithm can be applied to SAR images for detecting and matching stable features, particularly at lower scale levels, where the influence of speckle noise is reduced.

GCP extraction schemes using remotely sensed imagery based on the SIFT algorithm have also been developed. For example, Shargai et al. [7] utilized the algorithm to extract tie-points from aerial images, obtaining satisfactory results, although the details were not presented. Wessel et al. [8] developed the GCP extraction technique for near-real-time SAR images by using the SIFT algorithm in combination with DEM data and high-precision orbit parameters from a reference image to obtain GCPs, where a least-squares adjustment of the imprecise orbit is applied to eliminate incorrect matches. In the method proposed by Liu and Yu [9], the SIFT algorithm is used to match the destination and reference images, after which edge extraction is performed on SAR images by using the Canny operator. Liu et al. [10] proposed SIFT-based automatic tie-point extraction for multi-temporal SAR images, where a histogram-based preprocessing method is used to increase the number of tie-points, and SIFT parameters are optimized by extending the region of the descriptor.

This paper is arranged as follows, Section I outlines the application of the proposed method to GCP extraction of multi-temporal polarimetric SAR images acquired using various satellites at different viewing angles. It is found that, for the fully polarimetric SAR images GCP extraction, the locations and numbers of GCPs are different for each type of polarization data (HH, HV, VH or VV). Therefore, GCP extraction using TP images rather than each polarization image is suggested. In Section II, SIFT algorithm is described, after which the method for automatic GCP extraction of multi-temporal polarimetric SAR images is presented. In Section III, the characteristics of the areas used in the experiments are introduced. The experimental results are reported and discussed in Section IV. Finally, the paper is concluded in Section V.

\section{METHOD}

The proposed method for automatic GCP extraction of multi-temporal polarimetric SAR images is depicted the Fig. 1. The method starts with the base $\left(H H_{b}, H V_{b}, V H_{b}\right.$, and $\left.V V_{b}\right)$ and the warp $\left(H H_{w}, H V_{w}, V H_{w}\right.$, and $\left.V V_{w}\right)$ of the polarimetric SAR images. In addition, the total power received by each of these channels of the polarimetric radar system is considered to encapsulate all the polarizations. The following subsections outline each step of the method. 


\section{A. Automatic GCP extraction procedure}

\section{Keypoint Extraction}

To extract the candidate keypoints, the Scale-Invariant Feature Transform (SIFT) algorithm introduced by Lowe [6] is used. An outline of the pertinent points of the SIFT algorithm is presented below. The first step in detecting keypoints is the convolution of the image with Gaussian filters at different scale levels (referred to as scale-space extrema detection), follows by the generation of Difference-of-Gaussian (DoG) images from the differences between adjacent blurred images. If the scale space of an image is

$$
L(x, y, \sigma)=G(x, y, \sigma) * I(x, y),
$$

where

$$
G(x, y, \sigma)=\frac{1}{2 \pi \sigma^{2}} e^{-\left(x^{2}+y^{2}\right) / 2 \sigma^{2}}
$$

then the DoG image is given by

$$
D(x, y, \sigma)=(G(x, y, k \sigma)-G(x, y, \sigma)) * I(x, y)
$$

Here, $G(x, y, \sigma)$ is a variable-scale Gaussian, $I(x, y)$ is the input image, $\sigma$ is the scale level such that $k \sigma$ is a neighboring scale level, and

* is the convolution operator in $x$ and $y$.

Keypoints are identified as local maxima and minima in DoG images across all scale levels. Each pixel in a DoG image is compared with its eight neighbors at the same scale level and the nine corresponding pixels at the adjacent scale levels. If the pixel is a local maximum or minimum, it is selected as a candidate keypoint. In the second step, each candidate keypoint is checked for localization accuracy (keypoint localization). Since a close fit to nearby data in terms of location, scale level and ratio of principal curvatures is required, any keypoints which are found to have low contrast (i.e., high sensitivity to noise) or which are poorly localized along an edge are eliminated. The third step involves the assignment of an orientation to each keypoint. A gradient orientation histogram is computed in the neighborhood of the keypoint, and the contribution of each neighboring pixel is weighted by both the gradient magnitude and a Gaussian-weighted circular window with a value of $\sigma$ that is 1.5 times that of the scale level of the keypoint. Peaks of the histogram correspond to dominant orientations. The gradient magnitude $m(x, y)$ and the gradient orientation $\theta(x, y)$ are given by

$$
\begin{aligned}
& m(x, y)=\sqrt{(L(x+1, y)-L(x-1, y))^{2}+(L(x, y+1)-L(x, y-1))^{2}}, \\
& \theta(x, y)=\tan ^{-1}((L(x, y+1)-L(x, y-1)) /(L(x+1, y)-L(x-1, y))) .
\end{aligned}
$$

In the fourth step, the keypoint descriptor is computed as a set of orientation histograms on $4 \times 4$-pixel neighborhoods, with eight orientation bins in each histogram. Therefore, a SIFT descriptor is a 128-element feature vector. This vector is normalized to obtain a highly distinctive keypoint and to enhance the invariance to affine changes in illumination. 
Schwind et al. [1] proposed a modification of the original SIFT algorithm, which was named SIFT-OCT (where the first scale-space octave is skipped) as an extension for the SAR images. Images acquired with the spaceborne SAR sensors Radarsat-1 and ERS-2 were tested. They found out that matches with poor quality were generated in the first octave (highest scale level) of the scale space pyramid. However, more robust keypoints can be detected at lower scale levels due to the reduced influence of speckle noise at smaller scale levels in all octaves. Therefore, skipping the first octave of the scale space pyramid was suggested. Since in this study we focus on polarimetric SAR images, the extension of SIFT-OCT to polarimetric data was examined.

\section{Two-way Keypoint Matching}

Beis and Lowe [11] proposed an approximation algorithm referred to as Best-Bin-First (BBF) for matching SIFT keypoints. In the experiments by Schwind et al. [1], it was found that the performance of SIFT algorithm detection in combination with BBF matching is the highest for images acquired with the same sensor. However, the matching performance may be lower for images acquired from various satellites at different viewing angles. Therefore, the keypoint matching strategy proposed by Lowe [12] can be applied in order to achieve accurate GCP extraction of two or more images acquired from different viewpoints or with different sensors.

Dare et al. [3] also proposed an improved model for automatic feature-based image registration of SAR and SPOT images, in which they focused on developing a method for locating GCPs within the images. In this model, two-way feature matching algorithms are highlighted to ensure that similar features can be detected from different remote sensing imagery. Two-way matching was proposed since the redundancy is an important factor in the matching procedure. Redundancy can ensure that accurate matches are selected properly, with minimum improbable matches.

In the proposed method, the two-way keypoint matching is introduced in the keypoint matching procedure. This idea employs the initial keypoint matching procedure as proposed by Lowe [12], where the Euclidean distance between the descriptor vectors is calculated in order to match two descriptors. Matching the descriptor vectors in the base image (or input image) on the basis of the ratio of the descriptor distance between the two best matches of the warp image (or reference image) is performed for each keypoint. If the Euclidean distance of the second-closest match is smaller than the matching threshold, which is 0.6 times the distance of the closest match, the point is accepted as a "match for the base image" (referred to as forward matching), and a check is performed for all base images. This step is repeated by matching the feature descriptors in which the warp and base images are represented as an input image and a reference image, respectively. Using the same matching threshold, the matching points in this case are accepted as "matches for the warp image” (referred to as backward matching). Searching for corresponding points by forward matching will not produce the same results as by backward matching due to differences in the ratio between the two best matches. Finally, the intersections of these two matching sets are accepted as "two-way matches", and non-intersecting matches are eliminated. Thus, the two-way keypoint matching step excludes improbable correspondence keypoints. 


\section{RMSE Minimization}

To ensure that no false matches remain, the Root Mean Squared Error (RMSE) minimization is used. This step helps to remove large errors from the set of GCPs. First, the number of extracted GCPs is used to conduct the pseudo-affine transformation given in Eq. (6), and the RMSE is calculated for each point. Then, the GCPs with large errors are removed by applying a fixed threshold. The thresholds used in this experiment were common for all studied areas, and their values were fixed at 1.75 pixels through empirical examination in the case of data acquired from similar viewing angles. In the same manner, 5 and 1,700 pixels were used as thresholds for the cases of data acquired at different viewing angles and with different satellites, respectively. The pseudo-affine transformation is implemented to determine the predicted coordinates using the following equations:

$$
\begin{aligned}
& X_{i}^{\prime}=a_{1}+a_{2} x_{i}+a_{3} y_{i}+a_{4} x_{i} y_{i} \\
& Y_{i}^{\prime}=a_{5}+a_{6} x_{i}+a_{7} y_{i}+a_{8} x_{i} y_{i}
\end{aligned} .
$$

where $x_{i}$ and $y_{i}$ are the input source coordinates, $X_{i}$ ' and $Y_{i}$ ' are the predicted coordinates and $a_{1}$ to $a_{8}$ are polynomial coefficients.

\section{Information Entropy and Spatial Dispersion Quality Constraints}

To quantify the spatial distribution of GCPs, the information entropy of local regions and the spatial dispersion quality constraints are used. Cheng et al. [13] stated that although the number of points is not important, the robustness and the accuracy of GCP extraction are related to the distribution of the points. Hence, a broad distribution of points across the images is crucial. Taking the local regions as Regions Of Interest (ROI), with the GCPs at the respective centers of the regions, the entropy is derived from

$$
H_{i}=-\sum_{j=0}^{255} P_{j} \log _{2} P_{j}
$$

where $H_{i}$ is the entropy of local region $i, j$ is the grayscale intensity value (0-255) and $P_{j}$ is the probability of $j$ being within the ROI.

The weighted mean center $\left(\bar{x}_{w m c}, \bar{y}_{w m c}\right.$ ) is the average of the $x_{i}$ and $y_{i}$ coordinates for a series of points $i$ weighted by the entropy of the local region:

$$
\left(\bar{x}_{w m c}, \bar{y}_{w m c}\right)=\left(\frac{\sum_{i=1}^{n} w_{i} x_{i}}{\sum_{i=1}^{n} w_{i}}, \frac{\sum_{i=1}^{n} w_{i} y_{i}}{\sum_{i=1}^{n} w_{i}}\right) .
$$

Here, $x_{i}$ and $y_{i}$ are the coordinates of the point of interest, $w_{i}$ is the weight of point $i$, and $n$ is the number of points.

Therefore, an index Disp [13], which expresses the quality of spatial dispersion (where a small Disp value indicates poor spatial distribution and vice versa), is defined as 


$$
\operatorname{Disp}=\sqrt{\frac{\sum_{i=1}^{n}\left(x_{i}-\bar{x}_{w m c}\right)^{2}+\sum_{i=1}^{n}\left(y_{i}-\bar{y}_{w m c}\right)^{2}}{n}},
$$

A sufficient number of accurate GCPs are selected by referring to the information entropy of the local regions and spatial dispersion quality constraints. For the experiments, a $3 \times 3$ kernel is used for local regions that neighbor the GCPs. Further to define the number of GCPs within an image, the maximum value of Disp is selected, and each image should contain at least 15 points. In fact, in the experiments, three criteria are used to check whether the minimum matching requirements are met:

1) From [14], the minimum number of GCPs required is $n_{\min }=(t+1)^{*}(t+2) / 2$, where $t$ is the degree of the polynomial equation. An $n$-point pseudo-affine transformation (first-degree polynomial warp) is used.

2) From the experiments, the number of GCPs remaining after minimizing the RMSE is examined. It was found that more than 15 GCP matches remained in all cases.

3) The image size is considered in defining the minimum number of GCPs.

This method uses the SIFT algorithm for SAR imagery; however, the SIFT algorithm was originally developed for optical images. Since speckle noise in SAR images can lead to false detections at small scale levels, filtering is often used to remove noise [15], even though it may reduce the spatial resolution of the original image and decrease the number of detected keypoints. Furthermore, as in the original SIFT algorithm as proposed by Lowe, Random Sample Consensus (RANSAC) [16]—which is an iterative method capable of estimating the parameters of a mathematical model from a set of observed data even if it contains a large proportion of outliers-is used to remove false matches. Outliers are data that do not fit the model and arise from extreme levels of noise or from erroneous measurements. However, some disadvantages are found when using this method, such as the fact that no upper bound exists on the time necessary to compute these parameters. Furthermore, when the number of computational iterations is limited, the generated solution may not be the optimal result. From Eqs. (10) and (11), a larger number of iterations $(N)$ can increase the probability $p$ of producing a reasonable model. Let $u$ represent the probability that the obtained data point is an inlier and $v$ represent the probability of observing an outlier. A minimum of points $m$ are required to determine the underlying model parameters:

$$
\begin{gathered}
1-p=\left(1-u^{m}\right)^{N}, \\
N=\frac{\log (1-p)}{\log \left(1-(1-v)^{m}\right)} .
\end{gathered}
$$

Other disadvantages of RANSAC include the requirement for setting problem-specific predefined thresholds in order to determine the number of points that fit the model, and the necessity to estimate the model parameters using all of the data not identified as outliers. With the limitations described above, the proposed method can be applied to compensate for the use of 
RANSAC and to eliminate the effects of speckle noise. As a consequence, a large number of keypoints are initially selected, and accurate matches are eventually obtained in a time- and cost-effective manner.

\section{B. Geometric calibration}

Rather than scanning a surface, radar systems turn from side-to-side and record the locations of objects by using the distance from the sensor to the object along the line of sight. An image collected by using this geometry is referred to as a slant range image. Such images exhibit a systematic geometric distortion in the range direction. At ground range, pixel size varies across the range direction due to the changing incidence angle, and this makes the image appear compressed in the near range.

Images from PALSAR Level 1.1 were used here as experimental data. This means that slant range geometry is provided at Level 1.1, whereas this is not the case for ground range geometry. Therefore, in this step, geometric calibration is used to correct the ground range geometry by converting the distances from the slant range to the ground range. Subsequently the slant range radar images are re-sampled with a fixed ground range pixel size by assuming a flat terrain. After obtaining images with a fixed ground range pixel size, the dispersed GCPs then conform to the adjusted ground range pixel size.

\section{Performance evaluation of GCP extraction}

Casu et al. [17] exploited the amplitude information of the SAR image pairs, acquired at different times to calculate the deformation time-series. This approach calculates a matching correlation surface (MCS) as the inner product between two oversampled SAR images to estimate the range and azimuth shifts for each identified pixel. They also found that the accuracy of this approach is on the order of 1/10th-1/20th of a pixel. In this regard, the MCS can be exploited to estimate the signal-to-noise ratio (SNR). The SNR can be used to evaluate the performance of the GCP extraction.

The MCS [17] is then achieved by computing between the amplitudes of the base and the warp images determined from the dispersed GCPs for each polarimetric image, given in Eq. (12). Considering the image sizes, the appropriate matching window surrounding each GCP or identified pixel $(i, j)$ used for computing the MCS was set to $31 \times 31$ pixel size.

$$
\operatorname{MCS}(i, j)=\frac{\sigma_{b w}}{\sigma_{b} \sigma_{w}},
$$

where $\sigma_{b w}$ is the covariance term. $\sigma_{b}$ and $\sigma_{w}$ are the standard deviation values for the base and warp amplitude images, respectively.

Thus, the SNR is estimated from Eq. (13). It is represented by the ratio of the correlation surface's peak to the mean of the surrounding value. Therefore, the SNR can be used as an indicator of the reliability and accuracy of the GCP extraction. The higher SNR value is used to examine the improvements of GCP extraction that can be applied to any polarization data. 


$$
S N R=\frac{\max \left(M C S^{2}\right)}{\frac{\left[\sum_{i=1, j=1}^{N, M} M C S^{2}(i, j) \mid-\max \left(M C S^{2}\right)\right.}{N M}} .
$$

where $i$ and $j$ are the element for the generic pixel of range and azimuth coordinates $(x, y) . N$ and $M$ are the image size dimensions along the range and azimuth directions, respectively.

\section{EXPERIMENTAL REGIONS}

The fully polarimetric SAR images used for experiments, cover four areas in Japan. Fig. 2 shows examples of VH-polarization PALSAR images for each of these areas, which were generated through azimuth compression of PALSAR Level 1.1 images with a 1/16 compression ratio. In the images of the Chiba region, some urban areas and Narita International Airport can be seen. Additionally, agricultural areas, in particular paddy fields, are widespread along the Tone River (left-to-right in the center of the image) and along the coast (right side). In the images of the Kyoto region, most of the area is covered by mountains, and Obama and Wakasa Bay can be seen in the northern part of the image. The Hokkaido images show urban areas surrounded by mountains and the Sea of Japan. Finally, the images of the Saitama/Tokyo region depict the Kanto Plain, a flat area where commercial, residential and agricultural areas are mixed. The properties of the full polarization data are displayed in Table 1. Furthermore, the properties of the multi-temporal Japanese Earth Resources Satellite-1 (JERS-1) SAR images obtained with the same satellite at similar viewing angles are shown in Table 3, for which scenes in the Ubon Ratchathani region are used. The imaged region consists of woodland (the Phou Xiang Thong National Biodiversity Conservation), rivers (the Mun River joins the Mekong River at Khong Chiam) and rural areas. The properties of SAR images obtained with the same satellite at different viewing angles (Osaka, Japan), as well as images obtained with different satellites at different viewing angles (Buri Ram, Thailand) are presented in Table 4.

\section{RESULTS AND DISCUSSION}

The method given in Section II was applied to GCP extraction of multi-temporal polarimetric SAR images acquired (1) with the same satellite at similar viewing angles, (2) with the same satellite at different viewing angles and (3) with different satellites at different viewing angles. The details of the results and a discussion are presented below.

\section{A. GCP extraction of SAR images obtained with the same satellite at similar viewing angles}

Figs. 3(a) and (b) show the GCPs extracted from images of the Kyoto area, where the GCPs are found on the entire base and warp images for $\mathrm{HH}$ polarization images before and after the spatial dispersion quality steps, respectively. Table 2 lists the detailed experimental results of GCP extraction for the four areas in Japan (Chiba, Kyoto, Hokkaido, and Saitama/Tokyo). To verify the applicability of the proposed method, the SNR in conjunction with the MCS, as described in Section II C was used as an indicator 
of the reliability and accuracy of GCP extraction. The number of GCPs is shown for the two-way keypoint matching step, which includes forward, backward and identical matching. The calculated SNR values for the GCPs indicate that this filter strategy reduce improbable matches, however SNR values were still low. Therefore, RMSE minimization step was used which can remove large errors in the process of selecting the candidate GCPs. As a result, the number of GCPs decreased and highly accurate GCPs were derived. Furthermore, the quality of distribution of the GCPs was also quantified by utilizing the information entropy and spatial distribution quality constraints (column: SDQ). The columns entitled "Number of GCPs-SDQ" and "SNR-SDQ" show the number of GCPs and the SNR values after applying the information entropy and spatial dispersion quality constraints, respectively. The maximum Disp value limited the number of extracted GCPs. In a few cases of Chiba, it was found that the SNR values slightly decreased, since the points with the high match rates were eliminated. Nevertheless, the effect of this phenomenon was small, and thus the method is still considered effective for selecting GCPs. SNR values calculated at each consequent step continuously increased for all cases of the polarimetric SAR and TP images. Moreover, the computation time for the processes was short, and was dependent on the number of keypoints to be matched.

When comparing the results for the four areas, no differences were found. However, there are certain factors that affect the accuracy, such as the size of the images and the characteristics of the topography. To evaluate the changes in accuracy resulting from different image sizes, the different sizes of the same SAR images were used (first with the original images and then with images compressed using a 1/16 azimuth compression ratio). This was done by applying the adapted SIFT-OCT algorithm to the polarimetric SAR imagery, as described in Section II A. The comparative results are in Table 2 and Fig. 4, respectively. These results shows that the application of the SIFT-OCT algorithm (reduction in size) entails higher accuracy at all steps and reduces the processing time. Considering the performance in the case of the SIFT algorithm, Schwind et al. [1], [2], found that the majority of false matches occurred at the first octave (highest scale) of the scale space pyramid. Furthermore, the presence of speckle noise in SAR images obstructs accurate detection. For this reason, the GCP matching accuracy was poor in this case. On the other hand, considering the case of the SIFT-OCT algorithm for polarimetric SAR images compressed using a 1/16 azimuth compression ratio (Fig. 4), more robust keypoints can be found at the lower scale level due to the reduced influence of speckle noise in all the octaves with the compressed images. For this reason, accurate matches are selected, which results in high GCP matching accuracy. It can thus be seen that the SIFT-OCT algorithm can be effective in obtaining higher performance in GCP extraction of polarimetric SAR images.

In order to demonstrate the topographical factors, it is preferable to use SAR images with a 1/16 azimuth compression ratio, as noise is reduced in this way. Fig. 4(a) shows all polarizations for the Chiba area provide the highest GCP matching accuracy. The image of the Chiba area (partly flat/slightly fluctuating terrain), which exhibit a mixed type of land cover (an airport; various bodies of water; vegetation and conurbations). In the image of the Hokkaido area, the land cover constitutes a mixture of residential, 
agricultural and mountainous areas and various bodies of water. In fact, the accuracy of the polarizations for the Hokkaido area in Fig. 4(b) is lower than that of the Chiba area, owing to the mountains surrounding the imaged area. For this reason, the accuracy for all polarizations in the case of the Kyoto area (fluctuating terrain) in Fig. 4(c) is lower, as almost all of the images contain mountainous areas. The Saitama/Tokyo area, illustrates that in the case where the entire image consists of commercial and residential neighborhoods including high-rise buildings, all polarizations for the Saitama/Tokyo area in Fig. 4(d) yield the lowest GCP matching accuracy. The influence of low accuracy is attributed to the phenomena of shadows and the foreshortening effect, which occur at locations with buildings and are especially pronounced in densely built-up areas. In addition, from studying image of the Saitama/Tokyo area, it was demonstrated that the accuracy in neighborhoods consisting of high-rise buildings was lower than that for other residential areas in partly flat/slightly fluctuating terrain in Chiba or Hokkaido areas.

The performance of the GCP extraction with respect to polarimetric data in Table 2 and Fig. 4 was considered. It was found that the SNR values of the TP data were lower than those of polarized data in Table 2. This is because the influence of multiplicative speckle noise of the original SAR data (i.e., the proposed method was tested with the original images). The speckle noise influences the performance of the GCP extraction for SAR data. On the other hand, when the proposed method was tested with the same SAR images compressed using a 1/16 azimuth compression ratio, the speckle noise is reduced. It was found that the TP images generated the highest SNR values in all cases, as shown in Fig. 4. The graphical comparison of the four areas of the fully polarimetric data is shown in Fig 4. It can be observed that, in all cases, the TP images generated the highest SNR values (and hence the highest GCP matching accuracy). Therefore, GCP extraction performed by using a TP image, rather than each polarization image, maximizes the GCP matching accuracy for all polarization data. Considering the implications of this result, the TP is the sum of all of the polarization powers. Thus, TP images represent a combination of the characteristics of all four polarization images, and it follows that the GCPs retrieved from TP images encapsulate all four polarizations. Finally, as noted in Section III, the four areas include different types of land cover. Looking closely at the obtained results, no discrepancies are found in the GCP extraction images. Therefore, the method can function correctly regardless of the type of land cover.

In addition, this method is applicable to GCP extraction of multi-temporal SAR images acquired with the same satellite at similar viewing angles. Table 3 shows the results of GCP extraction for multi-temporal JERS-1 SAR images of Ubon Ratchathani area in Thailand. A total of 8 images were tested, where the image acquired on the earliest date (1993/03) was used as the base image, and the other images represented warp images. The results in Table 3 demonstrate that the proposed method performs well when applied to multiple images. Here, the GCP matching accuracy is high, with SNR values amounting to more than 0.01 in all cases. Finally, from this experiment on SAR images acquired with PALSAR and JERS-1, it can be considered that the method can be successful on other SAR images as well. 


\section{B. GCP extraction of SAR images obtained with the same satellite at different viewing angles and with geometric calibration}

PALSAR Level 1.1 images of an area of Osaka city (Fig. 5) obtained at different viewing angles were tested using the proposed method. Figs. 5(a) and (b) show the GCPs extracted from the base and warp images for HH polarization images before and after geometric calibration, respectively. Details of the test data are given in Table 4. The off-nadir angles of the base and warp images were $34.3^{\circ}$ and $23.1^{\circ}$, respectively. Only $\mathrm{HH}$ and $\mathrm{HV}$ polarization (and TP production) were tested, as the base images were taken with dual polarization. Fig. 5(a) presents the results of GCP extraction for the original HH data. It can be seen that the extracted GCPs span the entire area of the images. In addition, geometric calibration of the PALSAR Level 1.1 images was considered, as described in Section II B. To improve the GCP matching accuracy, geometric calibration of the SAR images was performed, and the results of GCP extraction for the geometrically calibrated HH data are presented in Fig. 5(b). Again, the extracted GCPs are spread across the entire area of the images. Table 5 lists the output from automatic GCP extraction for SAR images retrieved with the same satellite at different viewing angles. SNR values for each step in the method— namely, two-way keypoint matching, minimization of RMSE and application of spatial dispersion quality constraints—increase continuously before and after geometric calibration. This has been the case for both types of polarization including TP, which provided higher GCP matching accuracy after the calibration. Moreover, the SNR values before and after calibration are presented in Table 5, which shows the TP image generating the highest SNR value in both cases.

Considering Table 5 and Table 2, the TP data generated the highest SNR values in Table 5, while the SNR values of the TP data were lower than those of polarized data in Table 2. In both these cases, the original SAR images were used. To illustrate the contrast between images acquired at similar and different viewing angles, the Osaka city area (referred to as the "case with different viewing angles") was compared with the Saitama/Tokyo area (referred to as the "case with similar viewing angles"), since their topographies (urban land coverage) of the areas are comparatively similar. The $\mathrm{HH}, \mathrm{HV}$, and TP values in Table 2 (column: SNR-SDQ) for the Saitama/Tokyo area are 0.011, 0.030, and 0.029 pixels, and the values in Table 5 (column: SNR-SDQ) for the Osaka city area are $0.004,0.005$, and 0.014 pixels before geometric calibration and $0.005,0.007$, and 0.016 pixels after geometric calibration. For the Saitama/Tokyo area, it was found that the TP data provided higher SNR value than the HH data. However the SNR value of the HV data seemed to generate higher than the TP data. Because of the effect of the number of combined polarization data (i.e., the TP data for the Saitama/Tokyo area was produced from four polarization data, while the dual polarization (HH and HV) data were used to produce the TP data for the Osaka area). Since the difference is negligible (only 0.001), the TP data was still considered effective for generating the highest SNR value (or the highest GCP matching accuracy). It can be seen that the results in Table 5 were consistent with the results in Table 2 given in similar topographic characteristics.

In addition, Table 5 shows that the case with different viewing angles provided lower GCP matching accuracy at every step of the method. Even though the two-way keypoint matching presented in Table 2 (for the case with similar viewing angles) generated 
lower SNR values, by the final stage of the method, the case with similar viewing angles provided higher GCP matching accuracy. The lower accuracy in the case with different viewing angles is attributed to the influence of shadows and the foreshortening effect. These phenomena, which occur at locations with buildings and are especially pronounced in densely built-up areas, obstruct the analysis of SAR images. The Osaka city area is highly urbanized, with a large number of adjacent high-rise buildings. Moreover, the foreshortening effect increases as the incidence angle is reduced, as well as in the presence of high mountains (or high-rise buildings in this experiment). The off-nadir angles of the base and warp images are $34.3^{\circ}$ and $23.1^{\circ}$, respectively, for the SAR images obtained with the same satellite at different viewing angles. The incidence angles of the base and warp images were calculated based on the near-range and far-range angles, which are $36.5^{\circ}$ to $40.7^{\circ}$ and $24.8^{\circ}$ to $26.6^{\circ}$, respectively. Therefore, in the case with different viewing angles, the foreshortening effect can occur. This phenomenon may have an impact on the GCP extraction accuracy.

\section{GCP extraction of SAR images obtained with different satellites at different viewing angles}

GCP extraction of images of the Buri Ram area in the northeast part of Thailand retrieved from PALSAR Level 1.5 (represented as the base image) and JERS-1 Level 2.1 were tested, where the images were acquired at different viewing angles (Fig. 6). Details of the test data are listed in Table 4. The products of the PALSAR Level 1.5 images are available in ground range geometry, with multi-look processing in both the range and azimuth. The pixel spacing can be selected, and the latitudes and longitudes in the products can be calculated. For JERS-1 images acquired from the National Space Development Agency of Japan (NASDA), level 2.1 products were obtained by multi-look processing and were delivered in ground range projection with $12.5 \mathrm{~m}$ pixel spacing corrected for their specific antenna pattern and range spreading loss. Here, the polarization of the JERS-1 SAR data was HH only, and the PALSAR data incorporated dual polarization. The cases under examination were (1) HH PALSAR(base) and HHJERS-1(warp) and (2) HV PALSAR(base) and HHJERS-1(warp). Fig. 6 shows the GCP extraction results for HV PALSAR(base) and $H_{\text {JERS-1(warp), where the original }}$ data for the base and warp images are shown in Figs. 6(a) and (c), with red rectangles marking the overlap between the images. Figs. 6(b) and (d) highlight the GCP extraction from the two images. Table 6 also shows the GCP extraction results for these SAR images obtained with different satellites at different viewing angles. From this table, as in the other examples, SNR values increase after each step of the method for both the HH and HV polarizations. In general, the SNR of the result becomes higher when the number of measurements increases. The SNR was used as an indicator of the GCP extraction. As the SNR was computed based on the MCS, the MCS of all dispersed GCPs were computed using the amplitudes of the base and the warp images. A matching correlation factor was exploited for the performance evaluation of the GCP extraction. In some cases, it might be possible to obtain low MCS from some dispersed GCPs. Thus, low SNR value might be generated. Hence, it is not possible to always have higher SNR from large number of measurements while using the MCS based computation. In this regard, the GCP extraction of SAR images acquired at different sensors or different viewing angles generated lower SNR than those for the case of the same sensor or 
similar viewing angles. In addition, a rather low SNR values were obtained since only a small portion of the total area overlapped.

Comparing the results in Table 6 with those for the case when only the viewing angles were different (Table 5), the case of different satellites provided the lowest accuracy at each stage of the method. This is attributed to the differences in the technical specifications; namely, off-nadir angles, spatial resolution, swath width, acquisition dates (there is a period of around 9 years between the acquisition of the two sets of images), and the multi-look and gray-level characteristics. Since different sources acquire data based on these factors, different signal structures were produced. Hence, these factors had an impact on the detection of GCPs, and improbable matches were obtained. A final note about this experiment is that two combinations of polarization (HHPALSAR and $\mathrm{HH}_{\text {JERS-1}}, \mathrm{HV}_{\text {PALSAR }}$ and $\mathrm{HH}_{\text {JERS-1 }}$ ), were tested. It can be seen from Table 6 that GCP extraction with similar polarizations produced considerably higher SNR values than GCP extraction with different polarizations.

\section{CONCLUSIONS}

A method for increasing the reliability and accuracy of automatic GCP extraction for multi-temporal polarimetric SAR images was proposed. In this method, keypoints were extracted based on the SIFT algorithm, and two-way keypoint matching was used to reduce the number of improbable correspondence keypoints. To attain a high accuracy, the RMSE minimization was performed to remove any GCP candidates producing large errors. In the next step, only GCPs with high spatial dispersion were selected by using a filtering strategy based on information entropy and spatial dispersion quality constraints. Experiments showed that the computation time required by this method was short, as well as that the process of matching SAR images produces a high ratio of correct matches. Moreover, the SIFT-OCT algorithm was successfully extended to polarimetric SAR data. From the test results, it can be seen that TP images are most appropriate for GCP extraction from PolSAR data, which yields the highest SNR values. Furthermore, it is also reasonably applicable to dispersed GCPs extracted from data obtained using the other four polarizations. In addition, geometric calibration can be used to improve the GCP matching accuracy. By exploiting the amplitude information of a sequence of SAR images acquired at different times, the SNR in conjunction with the MCS can be used to properly evaluate the performance of GCP extraction. As a result, the proposed method can be successfully applied to achieve automatic GCP extraction of full scene multi-temporal polarimetric SAR images with different types of land cover that have been obtained with the same satellite at similar viewing angles, with the same satellite at different viewing angles, or with different satellites at different viewing angles. 


\section{REFERENCES}

[1] P. Schwind, S. Suri, P. Reinartz and A. Siebert, “Applicability of the SIFT operator to geometric SAR image registration,” International Journal of Remote Sensing, vol. 31, no. 8, pp. 1959-1980, Apr. 2010.

[2] S. Suri, P. Schwind, J. Uhl and P. Reinartz, "Modifications in the SIFT operator for effective SAR image matching," International Journal of Image and Data Fusion, vol. 1, no. 3, pp.243-256, Sep. 2010.

[3] P. M. Dare and I. J. Dowman, “An improved model for automatic feature based registration of SAR and SPOT image," ISPRS Journal of Photogrammetry and Remote Sensing, vol. 56, pp. 13-28, 2001.

[4] S. Kumar, K.V. Arya, V. Rishiwal and P. N. Joglekar, "Robust image registration technique for SAR images," ICIIS proceedings, IEEE Sri Lanka section, Aug. 2006.

[5] A. Rosenqvist, M. Shimada, N. Ito, and M. Watanabe, "ALOS PALSAR: A pathfinder mission for global-scale monitoring of the environment,” IEEE Trans. on Geosci. Remote Sens., vol. 45, no. 11, pp. 3307-3316, Nov. 2007.

[6] D. G. Lowe, “Distinctive image features from scale-invariant keypoints,” Int. J. Comput. Vis., vol. 60, no. 2, pp. 91-110, Nov. 2004

[7] Z. Shragai, S. Barnea, S. Filin, G. Zalmanson and Y. Doytsher, "Automatic image sequence registration based on a linear solution and scale invariant keypoint matching,” in Proc. of the ISPRS BenCOS workshop, Beijing, China, vol. 36, no. 3, Oct. 2005.

[8] B. Wessel, M. Huber, and A. Roth, "Registration of near real-time SAR images by image-to-image matching," in Proc. of Photogrammetric Image Analysis, Munich, Germany, pp. 179-184, Sep. 2007.

[9] J. Liu and X. Yu, "Research on SAR image matching technology based on SIFT," in Proc. 2st Int. Archives of Photogrammetry, Remote Sensing and Spatial Information Sciences, Beijing, China, vol. 37, part B1, pp. 403-408, Jul. 2008.

[10] L. Liu, Yu. Wang and Yi. Wang, "SIFT based automatic tie-point extraction for multi-temporal SAR images," in International Workshop on Education Technology and Training \& 2008 International Workshop on Geoscience and Remote Sensing, vol. 1, pp.499-503, 2008.

[11] J. Beis and D.G. Lowe, "Shape indexing using approximate nearest-neighbor search in high dimensional spaces," In IEEE Conference on Computer Vision and Pattern Recognition, San Juan, Puerto Rico, pp. 1000-1006, 1997.

[12] D.G. Lowe, “Object recognition from local scale-invariant features,” International Conference on Computer Vision ICCV, Corfu, pp. 1150-1157, 1999.

[13] L. Cheng, J. Gong, X. Yang, C. Fan, and P. Han, "Robust affine invariant feature extraction for image matching,” IEEE Geosci. Remote Sens. Lett., vol. 5, no. 2, pp. 246-250, Apr. 2008.

[14] J. Gao, "Digital analysis of remotely sensed imagery,” McGraw Hill Professional, New York, 645 pp, 2009.

[15] A. Lopes, R. Touzi, and E. Nezry, “Adaptive speckle filters and scene heterogeneity,” IEEE Trans. Geosci. Remote Sens., vol. 28, no. 6, pp. 992-1000, Nov. 1990.

[16] M. Fischler and R. Bolles, "Random sample consensus: A paradigm for model fitting with applications to image analysis and automated cartography," CACM, vol. 24, no. 6, pp. 381-395, Jun. 1981.

[17] F. Casu, A. Manconi, A. Pepe, and R. Lanari, "Deformation Time-Series Generation in Areas Characterized by Large Displacement Dynamics: The SAR Amplitude Pixel-Offset SBAS Technique”, IEEE Trans. Geosci. Remote Sens., vol. 49, no. 7, pp. 2752-27633, Jul. 2011.

Kamolratn Chureesampant received a B.S. degree in Computer Science Kasetsart University, Bangkok, Thailand, in 2004, a M.S. degree in Geographic Information Systems and Remote Sensing from the Asian Institute of Technology, Pathum Thani, Thailand, in 2006, and a Ph.D degree in Urban and Environmental Engineering from Kyoto University, Kyoto, Japan, in 2012.

Her main interests lie in the area of remote sensing image processing, particularly SAR and polarimetric SAR images for co-registration, classification and change detection applications.

Junichi Susaki (M’00) received B.S., M.S. and Ph.D. degrees in Civil Engineering from the University of Tokyo, Tokyo, Japan, in 1995, 1997 and 2000, respectively.

Since 2007, he has been an Associate Professor at Kyoto University, Kyoto, Japan. His current research interests include urban monitoring and modeling using SAR, optical sensors and LiDAR for applications such as disaster mitigation and environmental change analysis. 
Table 1: Properties of fully polarimetric SAR images acquired with the same satellite at similar viewing angles.

\begin{tabular}{|c|c|c|c|c|c|c|c|}
\hline Properties & \multicolumn{9}{c|}{ SAR images acquired with the same satellite at similar viewing angles } \\
(ALOS-PALSAR Level 1.1, Mode: PLR, Quad-pol)
\end{tabular}

Table 2: GCP extraction results for SAR images of the Chiba, Kyoto, Hokkaido and Saitama/Tokyo areas, Japan. The images were acquired with the same satellite at similar viewing angles for fully polarimetric SAR (HH, HV, VH and VV) images which include TP.

\begin{tabular}{|c|c|c|c|c|c|c|c|c|c|c|}
\hline \multicolumn{2}{|c|}{ Images } & \multicolumn{5}{|c|}{ Number of GCPs (points) } & \multicolumn{3}{|c|}{ SNR } & \multirow{3}{*}{$\begin{array}{c}\text { Computation } \\
\text { time (s) }\end{array}$} \\
\hline \multirow{2}{*}{ Area } & \multirow{2}{*}{ Polarization } & \multicolumn{3}{|c|}{ Two-way keypoint matching } & \multirow{2}{*}{$\begin{array}{c}\text { RMSE } \\
\text { minimization }\end{array}$} & \multirow{2}{*}{ SDQ } & \multirow{2}{*}{$\begin{array}{l}\text { Two-way } \\
\text { keypoint } \\
\text { matching }\end{array}$} & \multirow{2}{*}{$\begin{array}{c}\text { RMSE } \\
\text { minimization }\end{array}$} & \multirow{2}{*}{ SDQ } & \\
\hline & & Forward & Backward & Identical & & & & & & \\
\hline \multirow{5}{*}{ Chiba } & HH & 121 & 117 & 86 & 21 & 21 & 0.007 & 0.021 & 0.021 & 57 \\
\hline & HV & 404 & 416 & 310 & 63 & 49 & 0.002 & 0.011 & 0.012 & 70 \\
\hline & VH & 413 & 435 & 320 & 68 & 31 & 0.002 & 0.007 & 0.012 & 71 \\
\hline & VV & 106 & 117 & 75 & 25 & 20 & 0.004 & 0.014 & 0.009 & 52 \\
\hline & TP & 590 & 613 & 470 & 164 & 74 & 0.002 & 0.006 & 0.004 & 88 \\
\hline \multirow{5}{*}{ Kyoto } & HH & 360 & 358 & 289 & 79 & 21 & 0.001 & 0.004 & 0.011 & 82 \\
\hline & HV & 791 & 801 & 645 & 156 & 35 & 0.002 & 0.006 & 0.028 & 130 \\
\hline & VH & 769 & 764 & 614 & 154 & 154 & 0.002 & 0.005 & 0.005 & 128 \\
\hline & VV & 293 & 304 & 235 & 57 & 23 & 0.002 & 0.008 & 0.012 & 63 \\
\hline & TP & 213 & 217 & 181 & 77 & 31 & 0.002 & 0.004 & 0.009 & 66 \\
\hline \multirow{5}{*}{ Hokkaido } & HH & 349 & 356 & 275 & 52 & 20 & 0.001 & 0.004 & 0.013 & 89 \\
\hline & HV & 459 & 449 & 352 & 80 & 20 & 0.002 & 0.005 & 0.008 & 78 \\
\hline & VH & 449 & 446 & 339 & 58 & 55 & 0.002 & 0.008 & 0.009 & 80 \\
\hline & VV & 235 & 220 & 179 & 36 & 26 & 0.003 & 0.006 & 0.010 & 65 \\
\hline & TP & 831 & 838 & 675 & 221 & 26 & 0.002 & 0.002 & 0.005 & 145 \\
\hline \multirow{5}{*}{$\begin{array}{l}\text { Saitama/ } \\
\text { Tokyo }\end{array}$} & HH & 557 & 555 & 437 & 118 & 21 & 0.002 & 0.007 & 0.011 & 83 \\
\hline & HV & 616 & 599 & 475 & 99 & 27 & 0.002 & 0.006 & 0.030 & 90 \\
\hline & VH & 524 & 506 & 387 & 79 & 45 & 0.002 & 0.009 & 0.014 & 82 \\
\hline & VV & 697 & 719 & 553 & 148 & 125 & 0.001 & 0.006 & 0.008 & 95 \\
\hline & TP & 406 & 307 & 311 & 119 & 34 & 0.003 & 0.008 & 0.029 & 65 \\
\hline
\end{tabular}

$\mathrm{SDQ}=$ information entropy and spatial dispersion quality constraints

Table 3: GCP extraction results for multi-temporal SAR images of the Ubon Ratchathani area, Thailand. The images were acquired with the same satellite at identical viewing angles (JERS-1 Level 2.1; path-row: 126-277; size: 6,400 ×6,000; orbit direction: descending, off-nadir angle: $35^{\circ}$ ).

\begin{tabular}{|c|c|c|c|c|c|c|c|c|c|c|c|c|}
\hline \multirow[b]{3}{*}{ No. } & \multirow{3}{*}{$\begin{array}{c}\text { Acquisition } \\
\text { date } \\
\text { (yyy/mm/dd) }\end{array}$} & \multirow{3}{*}{$\begin{array}{c}\text { Image scene } \\
\text { (lat/long) }\end{array}$} & \multirow[b]{3}{*}{ Type } & \multicolumn{5}{|c|}{ Number of GCPs (points) } & \multicolumn{3}{|c|}{ SNR } & \multirow{3}{*}{ 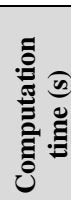 } \\
\hline & & & & \multicolumn{3}{|c|}{ Two-way keypoint matching } & \multirow[b]{2}{*}{$\begin{array}{c}\text { RMSE } \\
\text { minimization }\end{array}$} & \multirow[b]{2}{*}{ SDQ } & \multirow{2}{*}{ 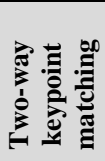 } & \multirow[b]{2}{*}{$\begin{array}{c}\text { RMSE } \\
\text { minimization }\end{array}$} & \multirow[b]{2}{*}{ SDQ } & \\
\hline & & & & Forward & Backward & Identical & & & & & & \\
\hline 1 & 1993/03/07 & $15.274 / 105.368$ & Base & & & & & & & & & \\
\hline 2 & $1995 / 05 / 08$ & $15.258 / 105.399$ & \multirow{7}{*}{ Warp } & 327 & 373 & 290 & 104 & 55 & 0.027 & 0.052 & 0.116 & 130 \\
\hline 3 & $1995 / 12 / 14$ & $15.258 / 105.381$ & & 314 & 298 & 246 & 59 & 27 & 0.029 & 0.124 & 0.197 & 134 \\
\hline 4 & 1996/01/27 & $15.528 / 105.392$ & & 780 & 765 & 627 & 189 & 54 & 0.015 & 0.052 & 0.166 & 130 \\
\hline 5 & $1996 / 03 / 11$ & $15.258 / 105.399$ & & 484 & 470 & 386 & 129 & 68 & 0.035 & 0.142 & 0.144 & 134 \\
\hline 6 & $1997 / 11 / 17$ & $15.258 / 105.404$ & & 180 & 165 & 138 & 44 & 22 & 0.072 & 0.128 & 0.202 & 131 \\
\hline 7 & $1998 / 02 / 13$ & $15.286 / 105.352$ & & 289 & 277 & 227 & 85 & 41 & 0.040 & 0.053 & 0.102 & 135 \\
\hline 8 & $1998 / 03 / 29$ & $15.258 / 105.423$ & & 489 & 487 & 391 & 102 & 23 & 0.027 & 0.089 & 0.120 & 132 \\
\hline
\end{tabular}

SDQ = information entropy and spatial dispersion quality constraints 
Table 4: Properties of SAR images acquired with the same satellite at different viewing angles and with different satellites at different viewing angles.

\begin{tabular}{|c|c|c|c|c|}
\hline Properties & \multicolumn{2}{|c|}{$\begin{array}{l}\text { SAR images acquired with the same satellite } \\
\text { at different viewing angles }\end{array}$} & \multicolumn{2}{|c|}{$\begin{array}{l}\text { SAR images acquired with different satellites } \\
\text { at different viewing angles }\end{array}$} \\
\hline Area & \multicolumn{2}{|c|}{$\begin{array}{l}\text { Osaka city, Japan } \\
\end{array}$} & \multicolumn{2}{|c|}{ Buri Ram, Thailand } \\
\hline Type & Base image & Warp image & $\begin{array}{c}\text { Base image } \\
\end{array}$ & Warp image \\
\hline Sensor type & ALOS-PALSAR Level 1.1 & ALOS-PALSAR Level 1.1 & ALOS-PALSAR Level 1.5 & JERS-1 Level 2.1 \\
\hline Frequency & $1.27 \mathrm{GHz}$ L-Band & $1.27 \mathrm{GHz}$ L-Band & $1.27 \mathrm{GHz}$ L-Band & 1.3 GHz L-band \\
\hline Mode or path-row (polarization) & FBD (Dual-pol) & PLR (Quad-pol) & FBD (Dual-pol) & $123-275(\mathrm{HH})$ \\
\hline Orbit direction & \multicolumn{2}{|c|}{ Ascending } & \multicolumn{2}{|c|}{ Descending } \\
\hline Acquisition date (yyyy/mm/dd) & $2009 / 07 / 16$ & $2009 / 05 / 09$ & 2007/09/01 & $1998 / 05 / 17$ \\
\hline Image center (lat/long) & $34.6042 / 135.5318$ & $34.7856 / 135.5171$ & $15.476 / 102.966$ & $15.258,102.646$ \\
\hline Size (sample $\times$ line) & $4,640 \times 18,432$ & $1,088 \times 18,432$ & $6,400 \times 5,700$ & $6,400 \times 6,000$ \\
\hline Off-nadir angle & $34.3^{\circ}$ & $23.1^{\circ}$ & $34.3^{\circ}$ & $35^{\circ}$ \\
\hline
\end{tabular}

Table 5: GCP extraction results for SAR images of the Osaka city area, Japan. The images were acquired with the same satellite at different viewing angles for dual polarimetric SAR (HH and HV) images which include TP.

\begin{tabular}{|c|c|c|c|c|c|c|c|c|c|c|}
\hline \multirow{3}{*}{$\begin{array}{l}\text { Geometric } \\
\text { calibration }\end{array}$} & \multirow{3}{*}{ Polarization } & \multicolumn{5}{|c|}{ Number of GCPs (points) } & \multicolumn{3}{|c|}{ SNR } & \multirow{3}{*}{$\begin{array}{c}\text { Computation } \\
\text { time (s) }\end{array}$} \\
\hline & & \multicolumn{3}{|c|}{ Two-way keypoint matching } & \multirow{2}{*}{$\begin{array}{c}\text { RMSE } \\
\text { minimization }\end{array}$} & \multirow{2}{*}{ SDQ } & \multirow{2}{*}{$\begin{array}{l}\text { Two-way } \\
\text { keypoint } \\
\text { matching }\end{array}$} & \multirow{2}{*}{$\begin{array}{c}\text { RMSE } \\
\text { minimization }\end{array}$} & \multirow[b]{2}{*}{ SDQ } & \\
\hline & & Forward & Backward & Identical & & & & & & \\
\hline \multirow{3}{*}{ Before } & HH & 187 & 136 & 123 & 68 & 52 & 0.0030 & 0.0034 & 0.0044 & 272 \\
\hline & HV & 270 & 223 & 176 & 89 & 67 & 0.0030 & 0.0039 & 0.0047 & 278 \\
\hline & TP & 144 & 97 & 87 & 51 & 50 & 0.0070 & 0.0132 & 0.0137 & 273 \\
\hline \multirow{3}{*}{ After } & HH & 187 & 136 & 123 & 69 & 53 & 0.0039 & 0.0044 & 0.0049 & 280 \\
\hline & HV & 270 & 223 & 176 & 92 & 68 & 0.0046 & 0.0064 & 0.0073 & 286 \\
\hline & TP & 136 & 77 & 67 & 32 & 32 & 0.0077 & 0.0155 & 0.0155 & 279 \\
\hline
\end{tabular}

$\mathrm{SDQ}=$ information entropy and spatial dispersion quality constraints

Table 6: GCP extraction results for SAR images of the Buri Ram area, Thailand. The images were acquired with different satellites at different viewing angles for similar polarizations and different polarizations.

\begin{tabular}{|c|c|c|c|c|c|c|c|c|c|}
\hline \multirow{3}{*}{ Scenario } & \multicolumn{5}{|c|}{ Number of GCPs (points) } & \multicolumn{3}{|c|}{ SNR } & \multirow{3}{*}{$\begin{array}{l}\text { Computation } \\
\text { time (s) }\end{array}$} \\
\hline & \multicolumn{3}{|c|}{ Two-way keypoint matching } & \multirow{2}{*}{$\begin{array}{c}\text { RMSE } \\
\text { minimization }\end{array}$} & \multirow{2}{*}{ SDQ } & \multirow{2}{*}{$\begin{array}{c}\text { Two-way keypoint } \\
\text { matching }\end{array}$} & \multirow{2}{*}{$\begin{array}{c}\text { RMSE } \\
\text { minimization }\end{array}$} & \multirow{2}{*}{ SDQ } & \\
\hline & Forward & Backward & Identical & & & & & & \\
\hline $\begin{array}{c}\text { HH }_{\text {PALSAR(base) }} \\
\text { and } \\
\text { HH JERS-1(warp) }^{\text {Jers }}\end{array}$ & 65 & 68 & 25 & 19 & 15 & 0.0021 & 0.0032 & 0.0042 & 175 \\
\hline $\begin{array}{c}\text { HV }_{\text {PALSAR(base) }} \\
\text { and } \\
\text { HH JERS-1(warp) }\end{array}$ & 94 & 80 & 47 & 33 & 16 & 0.0014 & 0.0022 & 0.0035 & 181 \\
\hline
\end{tabular}

SDQ = information entropy and spatial dispersion quality constraints

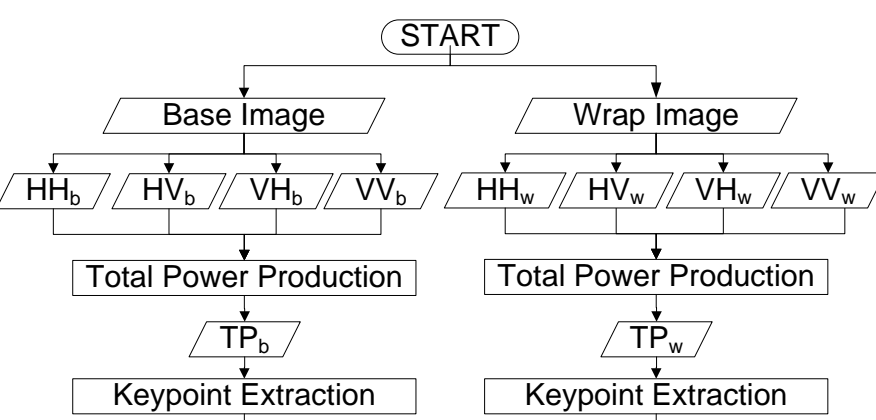

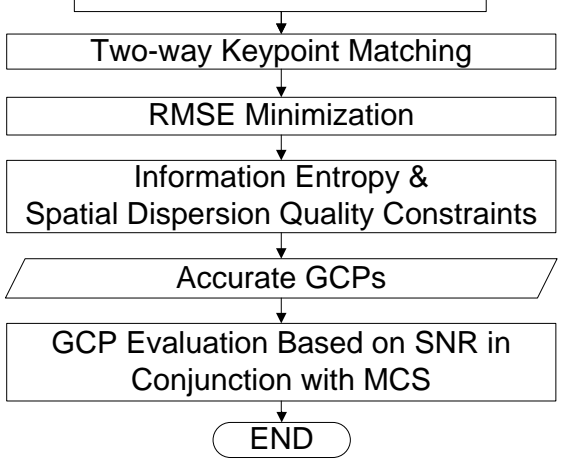

Fig. 1. Procedure for automatic GCP extraction.

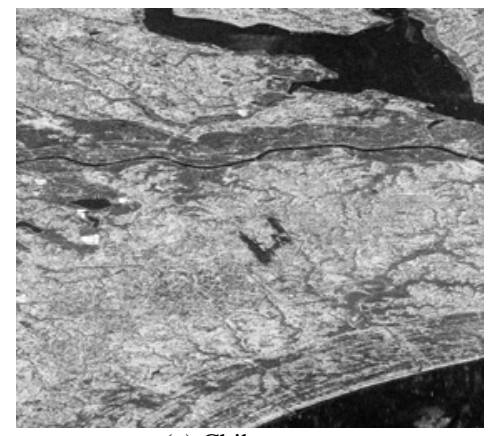

(a) Chiba area

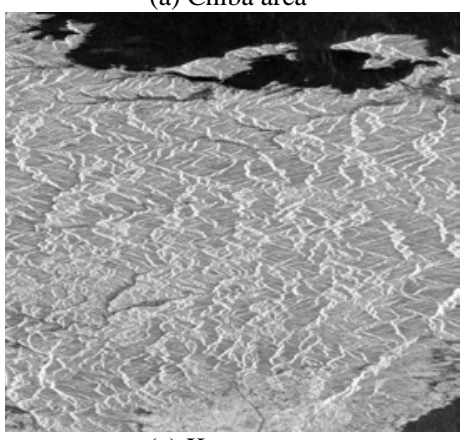

(c) Kyoto area

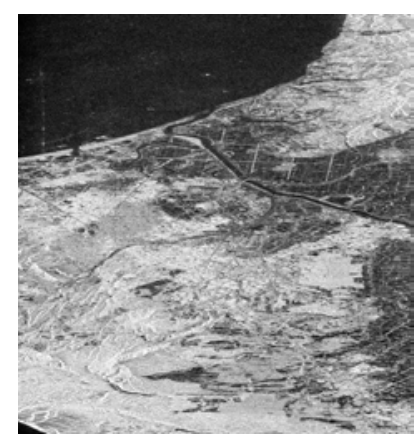

(b) Hokkaido area

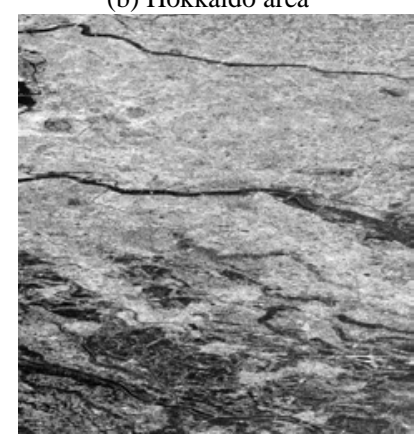

(d) Saitama/Tokyo area

Fig. 2. VH-polarization PALSAR images with 1/16 azimuth compression ratio for four investigated areas in Japan. 


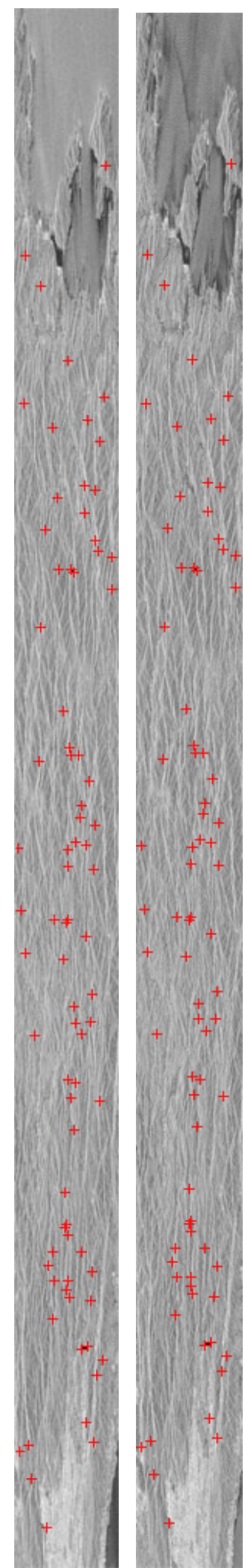

(a) $\mathrm{HH}_{\text {before } \mathrm{SDQ}}$

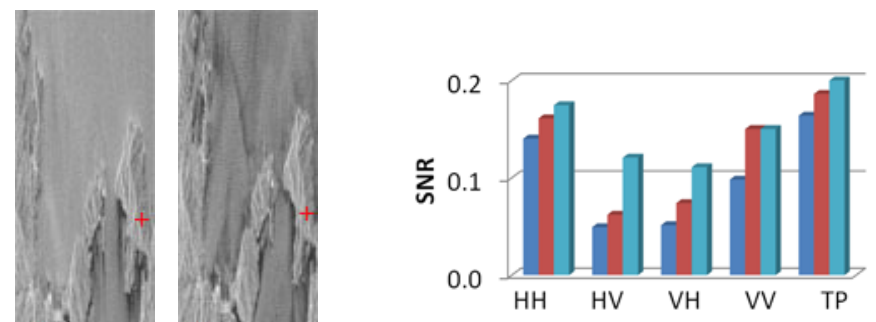

(a) Chiba area.

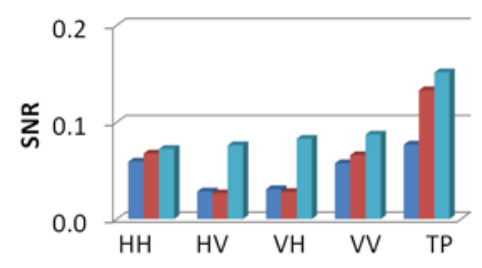

(c) Kyoto area.

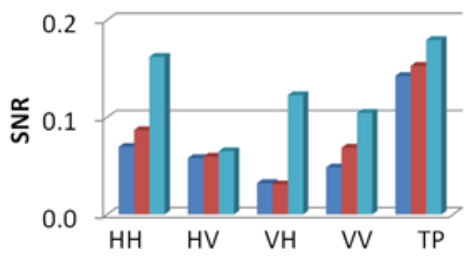

(b) Hokkaido area.

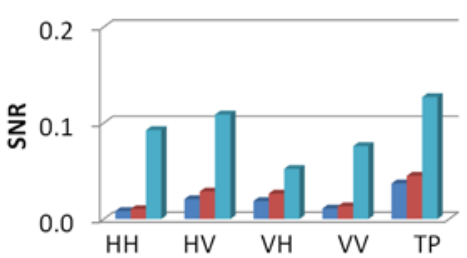

(d) Saitama/Tokyo area.

Two-way keypoint matching

RMSE minimization

SDQ

Fig. 4. GCP extraction results for SAR images acquired with the same satellite at similar viewing angles with the SIFT-OCT algorithm applied to fully polarimetic SAR images which include TP (1/16 azimuth compression ratio).

Fig. 3. GCP extraction from HH base and warp images (using original images with a size of $1,248 \times 18,432$ pixels) for the Kyoto area. GCPs are indicated by red crosses. The number of GCPs before and after applying information entropy and spatial dispersion quality constraints (SDQ) are 79 and 21, respectively. 


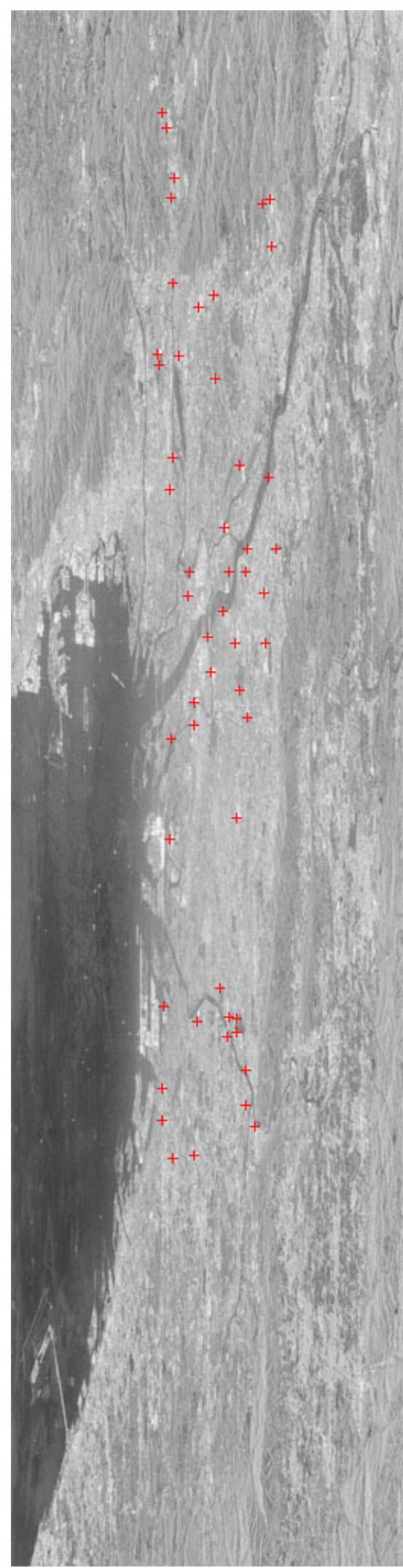

(a) $\mathrm{HH}_{\text {before geometric calibration. }}$

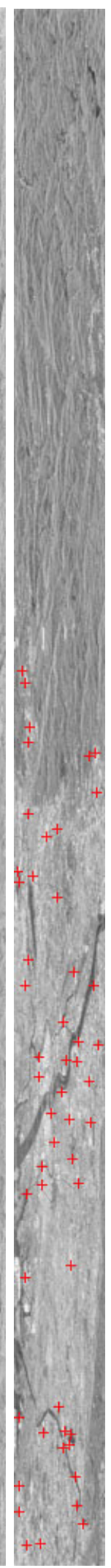

Fig. 5. GCP extraction from HH base and warp images before and after geometric calibration for the Osaka city area. GCPs are indicated by red crosses. The images were acquired with the same satellites at different viewing angles.

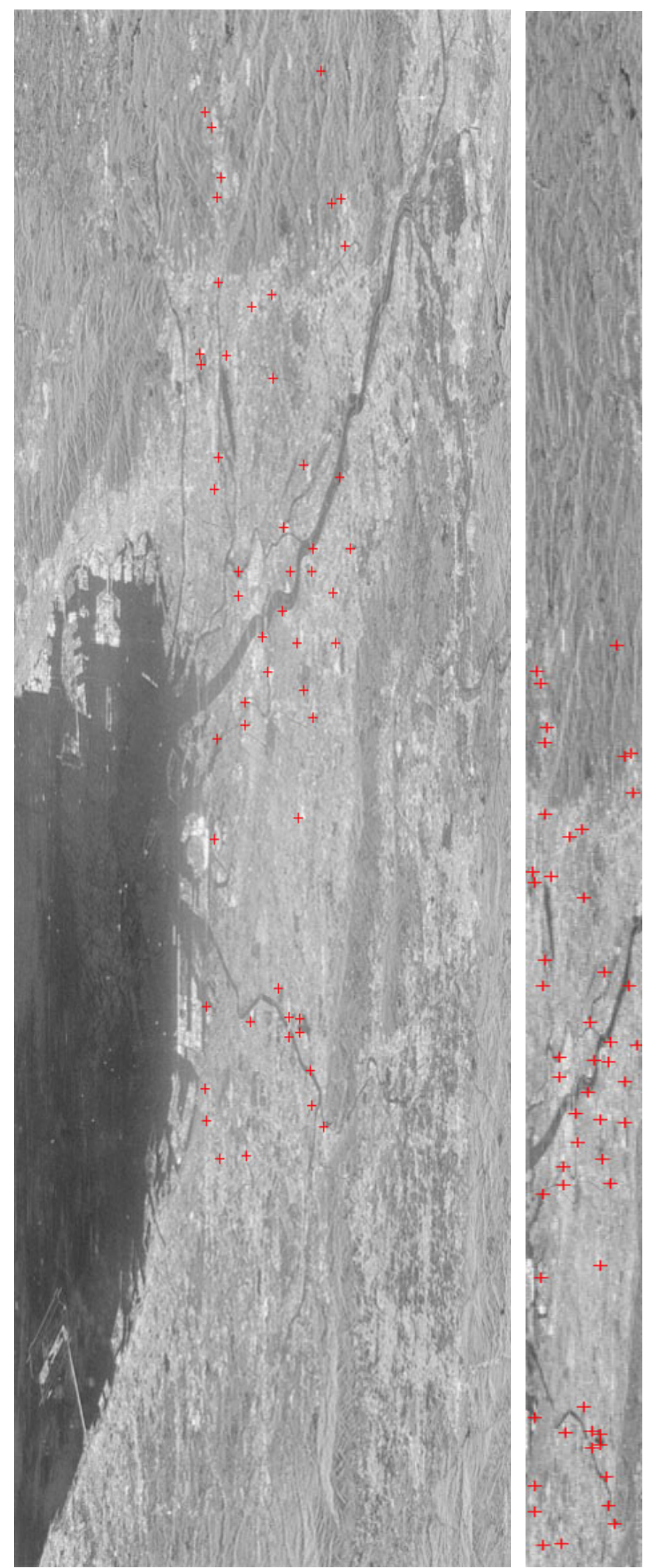

(b) $\mathrm{HH}_{\text {after geometric calibration. }}$ 

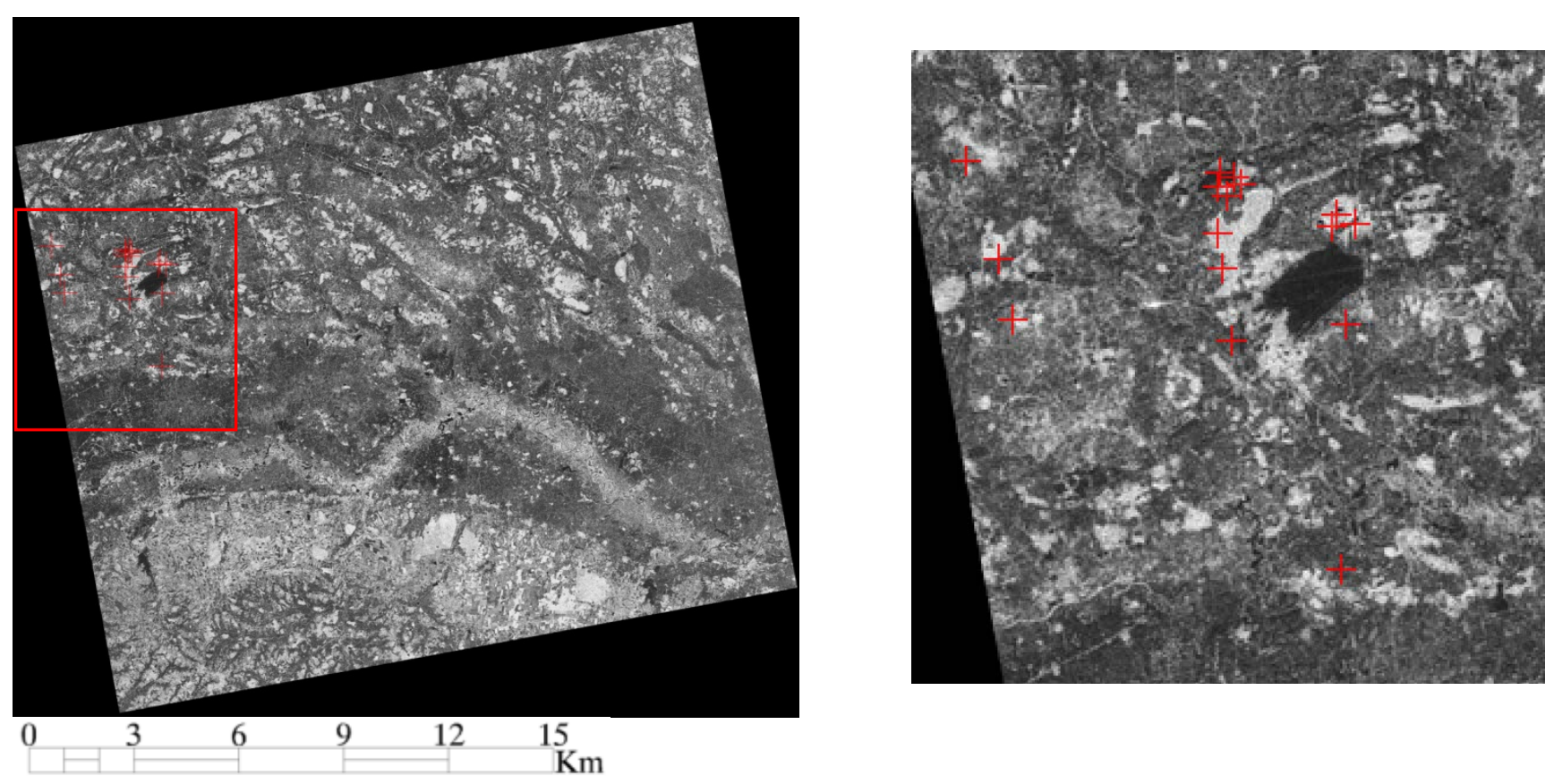

Map Scale 1:300,000

(a) Original ALOS-PALSAR (HV) image.

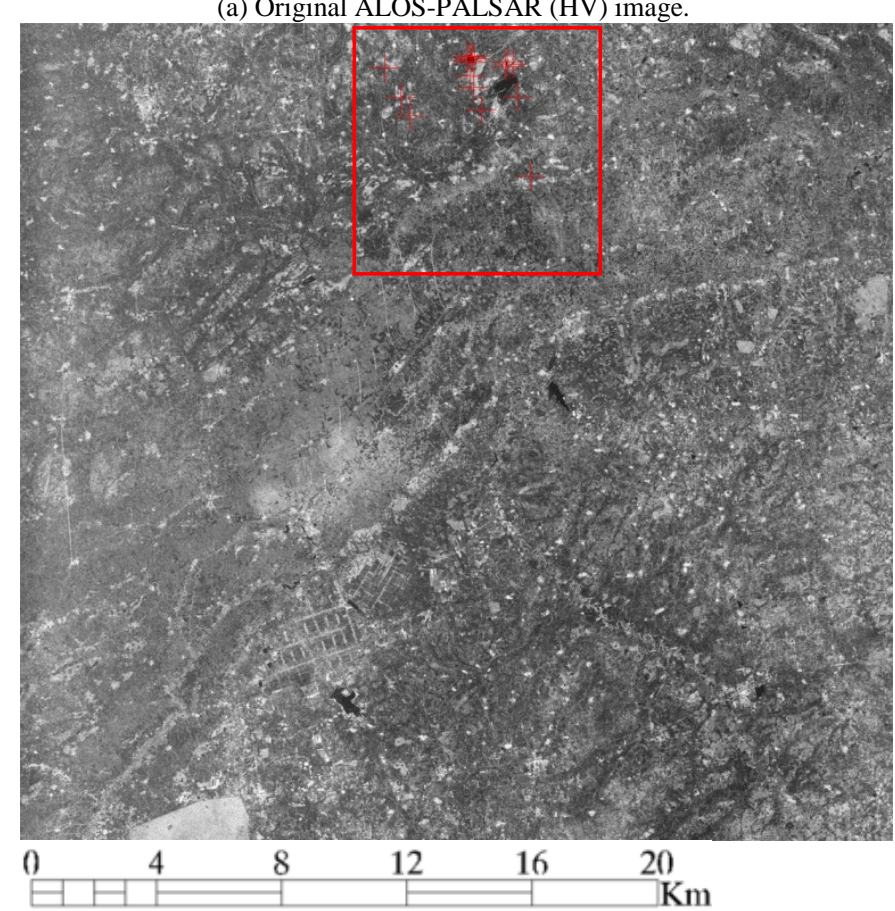

Map Scale 1:350,000

(c) Original JERS-1 (HH) image. (b) Magnified ALOS-PALSAR image with extracted GCPs.

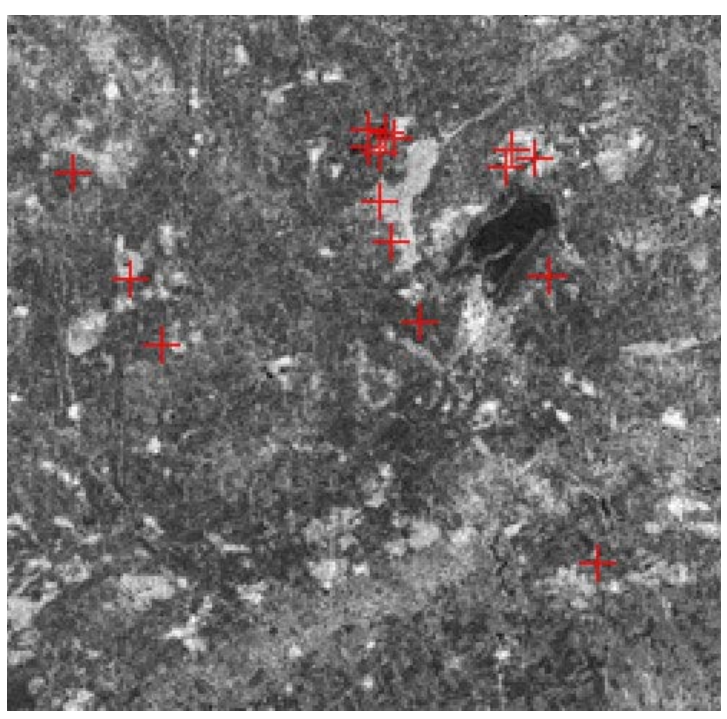

(d) Magnified JERS-1 image with extracted GCPs.

Fig. 6. GCP extraction with different polarizations for the Buri Ram area. The images were acquired with different satellites at different viewing angles. 